\title{
How a Patient Personalised Clinical Pharmacy Programme Can Secure Therapeutic Care in an Orthogeriatric Care Pathway (5P Project)?
}

\author{
Marine Barral, ',* Julie Martin, ',* \\ Emmanuelle Carre, ${ }^{2}$ Audrey Janoly- \\ Dumenil, ${ }^{3-5}$ Florence Ranchon, ${ }^{2,4,6}$ \\ Stéphanie Parat, ${ }^{2}$ Catherine Rioufol, ${ }^{2,4,6}$ \\ Sylvain Goutelle, 4,7,8 \\ Laurent Bourguignon, ${ }^{4,7,8}$ \\ Teddy Novais, ${ }^{4,9} 10$ Sebastien Doh, ${ }^{\prime \prime}$ \\ Matthieu Malatray, ${ }^{12}$ Philippe Chaudier, ${ }^{13}$ \\ Jerome Gauthier, ${ }^{14}$ Christine Pivot, ${ }^{3}$ \\ Christelle Mouchoux, ${ }^{4,9,15}$ \\ Delphine Hoegy (ID) $1,4,5$ \\ On behalf of 5P Study Group
}

'Pharmacie, Hospices Civils de Lyon, Lyon, France; ${ }^{2}$ Pharmacie Hôpital Lyon Sud, Hospices Civils de Lyon, Pierre-Bénite, France; ${ }^{3}$ Pharmacie Hôpital Edouard Herriot, Hospices Civils de Lyon, Lyon, France; ${ }^{4}$ Institut des Sciences Pharmaceutiques et Biologiques, Univ Claude Bernard Lyon I, Univ Lyon I, Lyon, France; ${ }^{5} 6-E A ~ 4 I 29$ P2S Parcours Santé Systémique- Univ Claude Bernard Lyon I, Univ Lyon I, Lyon, France; ${ }^{6}$ EA3738, CICLY Centre pour l'Innovation en cancérologie de Lyon, Univ

Claude Bernard Lyon I, Univ Lyon I, Lyon, France;

${ }^{7}$ Pharmacie Hôpital Pierre Garraud, Hospices Civils de Lyon, Lyon, France; ${ }^{8}$ CNRS, UMR 5558 , Laboratoire de Biométrie et Biologie Évolutive, Villeurbanne, France; ${ }^{9}$ Pharmacie Hôpital des Charpennes, Hospices Civils de Lyon, Villeurbanne, France; ${ }^{10}$ Université Claude Bernard Lyon I, Research on Healthcare Performance (RESHAPE), INSERM UI 290, Lyon, France; " 'Service de Gériatrie Hôpital Edouard Herriot, Hospices Civils de Lyon, Lyon, France; ${ }^{2}$ Service de Chirurgie

Orthopédique Hôpital Edouard Herriot, Hospices Civils de Lyon, Lyon, France; ${ }^{13}$ Service de Chirurgie Orthopédique Hôpital Lyon Sud, Hospices Civils de Lyon, Pierre-Bénite, France; ${ }^{14}$ Service

d'anesthésie et réanimation Hôpital Lyon Sud,

Hospices Civils de Lyon, Pierre-Bénite, France;

${ }^{15}$ Lyon Neuroscience Research Center, INSERM

UI 028, CNRS UMR5292, Lyon, France

*These authors contributed equally to this work.

Correspondence: Delphine Hoegy Hôpital Edouard Herriot, Groupement Hospitalier Centre, Hospices Civils de Lyon, 5 Place d'Arsonval, Lyon, 69003, France

Tel +33472 I| $9 \mid 82$

Fax +33472 II 7876

Email delphine.hoegy@chu-lyon.fr
Background: A new model was developed for integrating a personalised clinical pharmacy programme (5P project) into the orthogeriatric care pathway.

Objective: To secure the therapeutic care of orthogeriatric patients.

Design and Setting: Prospective descriptive study in a multisite teaching hospital from June 2019 to January 2020.

Subjects: Patients aged $\geq 75$ years admitted for hip fracture.

Methods: A prescription review was performed for all patients at inclusion. Other clinical pharmacy activities (additional prescription review, pharmaceutical interviews, medication reconciliation) were dedicated to "high-risk" patients. Potential medication errors (ME), either pharmaceutical interventions (PI) or unintentional discrepancies (UID), were recorded. The potential clinical impact of PI was evaluated by a pluriprofessional expert panel using a validated tool.

Results: In the 455 patients included, 955 potential ME were detected, that is $\geq 1$ potential ME for 324/455 (71\%) patients. In acute care, 561 PI were formulated during prescription review for 440/455 (97\%) patients and 348/561 (62\%) were accepted by physicians. Medication reconciliation was performed for 213 patients, 316 UID were identified. In rehabilitation units, a second prescription review was performed for 112/122 (92\%) "highrisk" patients, leading to $61 \mathrm{PI}$. The clinical impact was evaluated for 519/622 (83\%) PI. A consensus was obtained for $310 / 519(60 \%)$ PI: $147 / 310(47 \%)$ were rated as having minor clinical impact, $138 / 310$ (45\%) moderate, 22/310 (7\%) major, 2/310 (0.6\%) vital, and 1/310 $(0.3 \%)$ null.

Conclusion: The 5P project secured the orthogeriatric care pathway by detecting a great number of potential ME, including PI mostly considered as having a significant clinical impact.

Keywords: hip fracture, frail elderly, pharmaceutical services, medication errors, clinical relevance

\section{Introduction}

Hip fractures mostly affect older people (annual incidence of 620,000 in Europe in 2010) and induce high morbidity and mortality (18-33\% one-year mortality rate), functional decline, and sometimes postoperative complications. ${ }^{1,2}$ Recently, several studies have reported the benefits on both short- and long-term clinical outcomes of a pluriprofessional and integrated care pathway for older people with hip fractures (orthogeriatric care), such as fewer confusion episodes and postoperative complications, as well as lower mortality. ${ }^{3-5}$ Orthogeriatric care aims at organising patient 
care management before and after surgery in order to optimise medical and paramedical assessments, discharge planning, and to initiate enhanced recovery after surgery. This approach improves the quality and security of care due to collaboration between healthcare professionals. ${ }^{3-5}$

Previous studies have found that clinical pharmacy activities prevent between 1.2 and 4.2 medication errors (ME) per patient especially in older patients. ${ }^{6-10}$ Clinical pharmacy activities consist of prescription reviews, medication reconciliation, and patient or caregiver pharmaceutical interviews. Prescription review consists of an analysis and optimisation of a patient's medication prescription considering medical and laboratory data, therapeutic objectives, and guidelines. During this activity, pharmaceutical interventions (PI), corresponding to "any action initiated by a pharmacist directly resulting in a change of the patient's management or therapy" according to Dooley et $\mathrm{al}^{11}$ could be addressed to physicians. Medication reconciliation is a standardised process based on an exhaustive medication history using multiple sources; it permits to share complete information between healthcare professionals, to analyse and justify any discrepancies between usual medications and any new prescriptions, and so to detect potential ME. Finally, various pharmaceutical interviews conducted with patients or caregiver exist: admission interviews are a major source of information for medication reconciliation; discharge interviews consist of pharmaceutical advice regarding discharge prescription and any therapeutic change during hospitalisation; and targeted pharmaceutical informative interviews consist of giving pharmaceutical advice on a specific medication.

Although their interest has been highlighted in geriatric and/or orthopaedic units, ${ }^{6,7,12,13}$ to our knowledge, there is no data regarding any clinical pharmacy programmes developed all along this care pathway. Thus, a new model was created for integrating a patient personalised clinical pharmacy programme (5P project) into the orthogeriatric care pathway. The main objective was to optimise and secure therapeutic care of orthogeriatric patients, by describing the clinical pharmacy activities performed and evaluating the potential clinical impact of PI using a validated tool.

\section{Materials and Methods}

The clinical pharmacy programme was developed and described by Hoegy et al. ${ }^{14}$ Briefly, a Delphi technique was used with surgeons, geriatricians, paramedics, and pharmacists involved in the orthogeriatric care pathway in order to define criteria for prioritization of patients based on adverse events, and to position clinical pharmacy activities at the most relevant steps of the pathway.

\section{Design and Setting}

A prospective study was carried out in a French multi-site teaching hospital over eight months, from June, 3rd 2019 until January, 31st 2020. Patients were recruited from four sites. Two of them had a surgical emergency department, orthopaedic or geriatric units, and rehabilitation units. The two others had only rehabilitation units.

\section{Patients and Levels of Risk}

Consecutive patients aged 75 or older admitted to the participating hospitals for hip fracture during the study period were included. These patients were divided in two groups by clinical pharmacists according to their level of risk of adverse event: "low-risk" (LR) or "high-risk" (HR). HR patients presented at least two of the following criteria: being aged $\geq 90$ years, being obese (body mass index $\geq 30$ ) or diabetic, being prescribed a potentially inappropriate medication (PIM) for older people (list defined in Laroche et $\mathrm{al}^{15}$ ), suffering from at least one cardiovascular disease. The level of risk of patients was regularly revaluated by checking their medical records for an intercurrent event (postoperative complications or newly diagnosed diseases), or upon medical or pharmaceutical request.

\section{Clinical Pharmacy Programme}

The $5 \mathrm{P}$ project is summarized in Figure 1. In acute care (post-surgery step), a prescription review was performed for all patients, either in orthopaedic or geriatric units. No additional clinical pharmacy activity was performed for LR patients. Medication reconciliation at admission and transfer/discharge was conducted for HR patients. Whenever possible, ie, absence of cognitive impairment or postoperative confusion, admission and/or discharge interviews with patients were performed.

In rehabilitation units, a second prescription review was performed for HR patients. If appropriate, a targeted pharmaceutical informative interview about oral anticoagulant and/or discharge interviews were proposed to eligible patients. For instance, HR inpatients initiating an oral anticoagulant treatment, or patients already treated with oral anticoagulants and requiring more information were eligible to a targeted informative interview. 


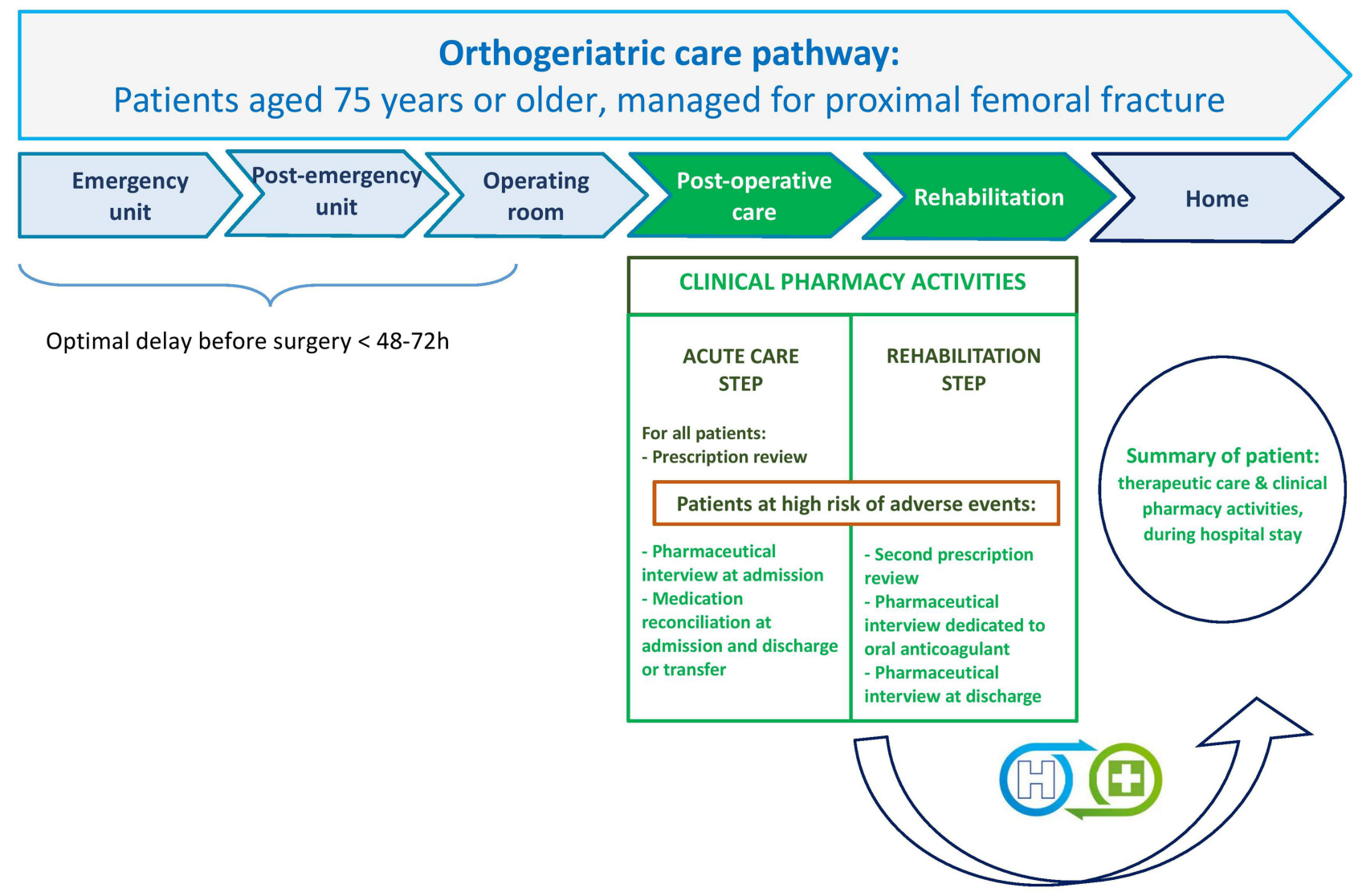

Figure I Personalised clinical pharmacy programme integrated into an orthogeriatric care pathway.

\section{Descriptive Analysis and Outcomes}

A descriptive analysis per protocol was performed for the following outcomes: number of inpatients who experienced at least one potential ME, which can be either PI during prescription review or unintentional discrepancies (UID) during medication reconciliation; number of potential ME detected (PI and/or UID); number of PI, and for each, the type of drug-related problem (DRP) and pharmacist recommendations according to the French Society of Clinical Pharmacy (SFPC) criteria, ${ }^{16}$ PI acceptance rate; number of UID at admission and at transfer/discharge; prescription modification rate following UID.

The potential clinical impact of PI was retrospectively assessed by a pluriprofessional expert panel composed of an anaesthetist, a geriatrician, a surgeon, and a pharmacist, using the clinical dimension of the CLinical, Economic, and Organizational (CLEO) tool (harmful, null, minor, moderate, major, vital, not determined; Table 1), developed and validated by Vo et al. ${ }^{16}$ The Economic and Organizational dimensions of this tool were not considered in the $5 \mathrm{P}$ project, consequently PI such as "non-conformity to the hospital formulary" (medications unavailable at the hospital) which were made essentially because of their organizational impact, were excluded. Firstly, PI were reviewed by two pharmacists (MB and EC). Secondly, PI were classified in "DRP and medication categories" by two pharmacists (JM and AJD). Thirdly, PI were rated by each expert independently. The pharmacist rating corresponded to a majority in opinion of individual ratings performed by five pharmacists (MB, EC, DH, AJD, and JM) independently. Finally, ratings from each expert were compared: the final rating was defined as "no consensus" (4 different ratings or in case of 2 times 2 identical ratings) or according to the majority rating ( $\geq 3$ identical ratings, or in case of 2 identical ratings and the 2 other ratings different).

\section{Data Collection}

Patient data (level of risk of adverse event, risk criteria, intercurrent event, and date of death) and their care pathway (origin before hospital admission, destination after discharge, and length of stay -LOS- in each unit) were collected from medical records. 
Table I Clinical Dimension of the CLEO Tool to Assess the Clinical Impact of Pharmaceutical Interventions

\begin{tabular}{|l|l|}
\hline $\begin{array}{l}\text { Clinical } \\
\text { Dimension }\end{array}$ & Description \\
\hline Harmful & Negative effect on patient in regard to clinical situation, knowledge, satisfaction, adherence, or quality of life \\
\hline Null & No effect on patient in regard to clinical situation, knowledge, satisfaction, adherence, or quality of life \\
\hline Minor & $\begin{array}{l}\text { Effect on patient in regard to clinical situation, knowledge, satisfaction, adherence, or quality of life OR damage, which does } \\
\text { not necessitate surveillance or treatment }\end{array}$ \\
\hline Moderate & Damage necessitates surveillance or treatment and does not lead to hospitalization or prolongation thereof \\
\hline Major & $\begin{array}{l}\text { Damage that leads to hospitalization or prolongation thereof OR } \\
\text { Damage that leads to disablement or impairment }\end{array}$ \\
\hline Vital & Damage that leads to intensive care treatment or death \\
\hline Not determined & The available information does not allow to determine the clinical impact \\
\hline
\end{tabular}

Notes: The clinical impact is evaluated for the patient's benefit. Harm: alteration of the physical and mental capacities arising from an accident or illness. Quality of life: physical function (autonomy, physical abilities, capacity to perform the tasks of daily life), psychological (anxiety, depression, emotion), social (relative to family environment, friendly or professional, engaging in personal relationships, participation in social and leisure activities) and somatic (symptoms related to the disease). Monitoring: monitoring clinically relevant (physiological or psychological), biological. Treatment: changing therapy or adding a medical/surgical treatment.

Data regarding clinical pharmacy activities were collected prospectively by each pharmacist. For prescription review, PI were rated according to the SFPC criteria. ${ }^{16}$ The name of the medication concerned by PI and the PI acceptance by physicians were recorded. For medication reconciliation, the number of medications prescribed before hospitalization, at admission, and discharge, and the number of UID were gathered. Any prescription modification by physicians was collected for UID.

\section{Statistical Analyses}

Statistical analyses were performed per protocol using SAS software version 9.4 (SAS Institute Inc., Cary, NC, USA). If clinical pharmacy activities were not performed, a dedicated implementation analysis was done by Martin et al (unpublished data). Categorical variables were expressed as frequency (percentage); continuous variables were expressed as mean \pm standard deviation (SD) or median (interquartile, [IQR]).

\section{Results}

\section{Patients and Care Pathway}

\section{Characteristics}

A total of 455 patients were included in the study, 284/455 (62\%) were considered as HR patients. Among them, 255/ 284 (90\%) were HR at inclusion, and 29/284 (10\%) patients were LR at inclusion and were reassessed as HR during orthogeriatric care pathway. A total of 202/455 (44\%) patients were $\geq 90$ years, $375 / 455(82 \%)$ had at least one cardiovascular disease, $82 / 455(18 \%)$ were either obese or diabetic, and 67/455 (15\%) received at least one PIM. Most HR patients met two risk criteria (204/255, 80\%), 44/255 (17\%) met three, and 7/255 (3\%) met all four criteria.

Most patients were admitted directly from home (272/ $455,60 \%)$, nursing homes $(126 / 455,28 \%)$, or healthcare facilities $(52 / 455,11 \%)$. In acute care, the median [IQR] LOS was 8 [6-12] days. A total of 191/455 (42\%) patients were transferred to $5 \mathrm{P}$ project rehabilitation units, their median [IQR] LOS was 35 [25-51] days. Among patients admitted to the hospital directly from home, 122/272 $(45 \%)$ were discharged home. A total of $36 / 455(8 \%)$ patients died during their hospitalization.

\section{Outcomes of 5P Project and Clinical Relevance of PI}

Regarding the 455 included patients, 955 potential ME (PI + UID) were detected, corresponding to a mean \pm SD of $2.1 \pm 2.5$ ME per patient, and at least one ME was noticed for 324/455 (71\%) patients. Regarding the $284 \mathrm{HR}$ patients, $755 \mathrm{ME}$ were detected, corresponding to a mean $\pm \mathrm{SD}$ of $2.7 \pm 2.8 \mathrm{ME}$ per patient, and at least one ME was noticed for 225/284 (79\%) HR patients.

\section{Clinical Pharmacy Activities in Acute Care (Orthopaedic or Geriatric Units)}

A total of 561 PI were made corresponding to 440 prescription reviews performed $(440 / 455,97 \%)$ for all patients, and at least one PI was made for 287/440 (65\%) 
Table 2 Clinical Pharmacy Activities and Outcomes in the Orthogeriatric Care Pathway

\begin{tabular}{|c|c|c|c|c|}
\hline & \multicolumn{2}{|c|}{ Acute Care, $n=455$ Patients } & \multirow{2}{*}{$\begin{array}{c}\text { Rehabilitation, } n=122 \\
\text { Patients }\end{array}$} & \multirow[t]{2}{*}{ Total } \\
\hline & At Admission & $\begin{array}{l}\text { At Transfer or } \\
\text { Discharge }\end{array}$ & & \\
\hline Prescription review & 440 & NA & 112 & 552 \\
\hline PI & 561 & NA & 61 & 622 \\
\hline Number of PI per patient, mean \pm SD & $1.3 \pm 1.3$ & NA & $0.5 \pm 0.8$ & NA \\
\hline Acceptance rate, $\mathrm{n} / \mathrm{N}(\%)$ & $348 / 56 \mid(62 \%)$ & NA & $40 / 61(66 \%)$ & $388 / 622(62 \%)$ \\
\hline Medication reconciliation & 213 & 105 & NA & 318 \\
\hline $\begin{array}{l}\text { Number of medications per patient, } \\
\text { mean } \pm \text { SD }\end{array}$ & $\begin{array}{l}\text { Before admission: } 8.0 \\
\quad \pm 3.7\end{array}$ & At discharge: $7.3 \pm 3.7$ & NA & NA \\
\hline UID & 316 & 17 & NA & 333 \\
\hline Number of UID per patient, mean \pm SD & $1.5 \pm 2.2$ & $0.2 \pm 0.5$ & NA & NA \\
\hline Acceptance rate, $\mathrm{n} / \mathrm{N}(\%)$ & $210 / 316(66 \%)$ & II/I7 (65\%) & NA & $221 / 333(66 \%)$ \\
\hline
\end{tabular}

Abbreviations: NA, not applicable; PI, pharmaceutical intervention; SD, standard deviation; UID, unintentional discrepancy.

patients. Medication reconciliation at admission was performed for 213/284 (75\%) HR patients, and identified 316 UID, and at least one UID was detected for 116/213 (54\%) patients (Table 2). Admission interviews were conducted with 107/284 (38\%) HR patients.

The most common medication classes identified by PI were nervous system medications (291/561, 52\%) including a majority of analgesics (morphine, acetaminophen ... etc.), medications for blood and blood-forming organs (83/561, $15 \%)$ including antithrombotic and infusion fluids, cardiovascular system medications $(65 / 561,12 \%)$, and medicinal products for the alimentary tract and metabolism (63/561, $11 \%)$. Most common DRP detected were "supratherapeutic dosages" $(90 / 561,16 \%)$, "non-conformity to guidelines" (80/561, 14\%), "absence of medications for a valid medical indication" (75/561, 13\%), and "non-prescription medications" (72/561, 13\%). Main pharmacist's recommendations were "addition of a medication" (145/561, 26\%), "dosage adjustment" (124/561, 22\%), or "medication discontinuation" $(123 / 561,22 \%)$.

\section{Clinical Pharmacy Activities in Rehabilitation Units}

Among the 122 patients rehabilitated in 5P project units, $112 / 122(92 \%)$ had a second prescription review, leading to $61 \mathrm{PI}$, and at least one PI was made for 42/112 (38\%) patients. The most frequent medication classes concerned by PI were similar to the ones in acute care: nervous system medications $(17 / 61,28 \%)$ including as well as analgesics (morphine or acetaminophen) and antidepressants (bromazepam or serotonin reuptake inhibitor antidepressant), medications for blood and blood-forming organs (13/61, 21\%) including antithrombotic and infusion fluids, and cardiovascular system medications (12/61, 20\%). Most common DRP detected were "non-conformity to guidelines" $(10 / 61,16 \%)$, "non-conformity to therapeutic hospital formulary" (9/61, 15\%), "supratherapeutic dosages" (9/ $61,15 \%)$, and "unjustified medications" (9/61, 15\%). Main pharmacist's recommendations were "medication switch" (19/61, 31\%), "dosage adjustment" $(15 / 61,25 \%)$, or "addition of a medication" (12/61, 20\%).

Targeted pharmaceutical informative interviews were conducted with 13/64 (20\%) HR patients treated with oral anticoagulants, 9/13 (69\%) had direct oral anticoagulants, and 4/13 (31\%) had anti-vitamin K anticoagulants.

\section{Clinical Pharmacy Activities at Discharge}

Medication reconciliation at discharge was completed for 105/213 (49\%) patients who benefitted from an admission medication reconciliation and identified 17 UID. At least one UID was detected for 11/105 (10\%) patients (Table 2).

Among them, 29/105 (28\%) had a discharge interview, either in acute or rehabilitation units.

\section{Potential Clinical Impact of PI}

The potential clinical impact of 519/622 (83\%) PI gathered in 127 "DRP and medication categories" was rated by the pluriprofessional expert panel (Figure 2). The 20 most common "DRP and medication categories" are presented in Tables 3 and 4, and correspond to 302/519 (58\%) PI. 


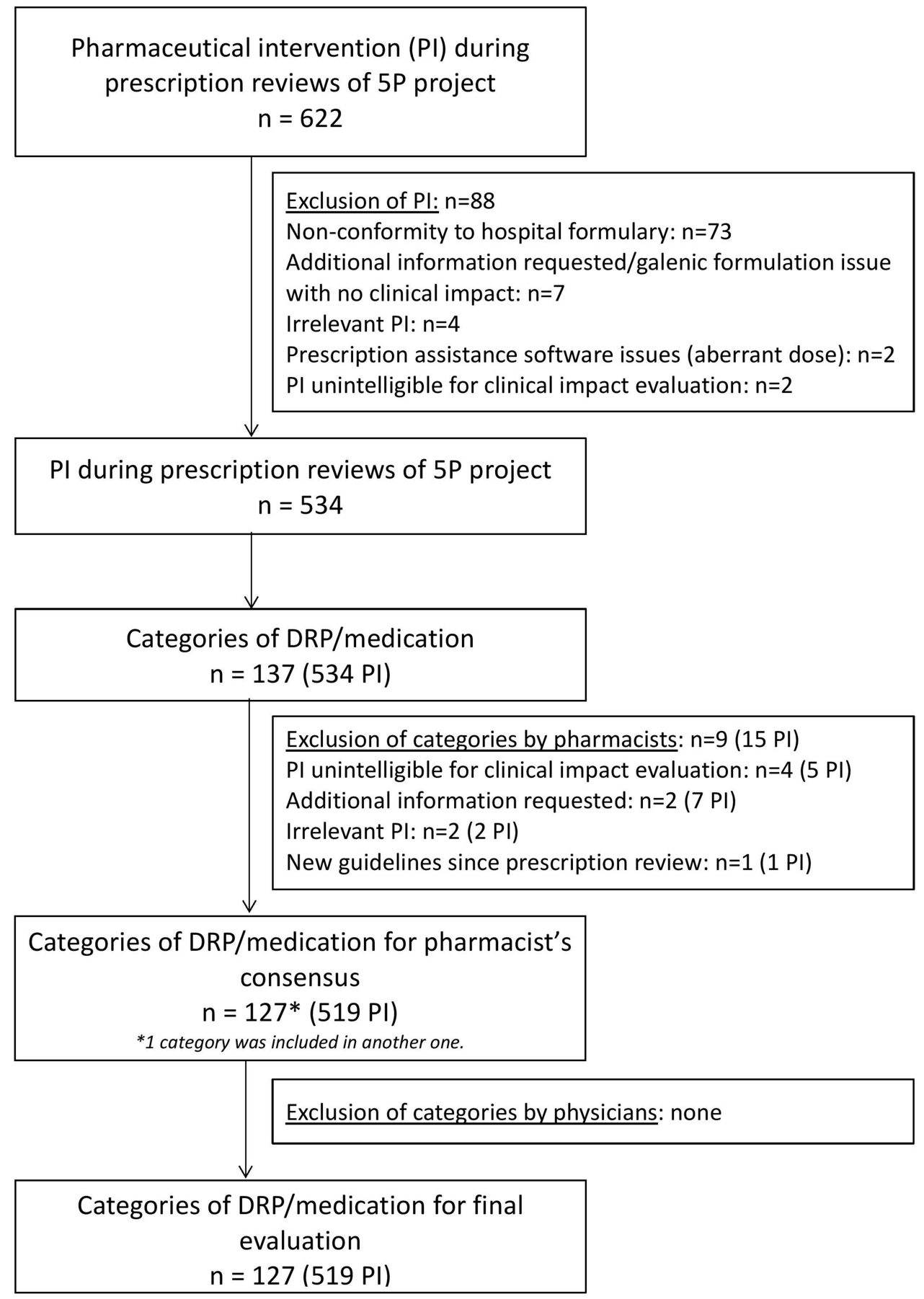

Figure 2 Flowchart of inclusion of PI and categories of PI for clinical impact evaluation by the pluriprofessional expert panel. Abbreviations: DRP, drug-related problem; PI, pharmaceutical intervention.

A consensus was obtained for 94/127 (74\%) "DRP and medication categories" corresponding to $310 / 519$ (60\%) PI.

Among "DRP and medication categories" for which a consensus was reach, the potential clinical impact was mainly minor $(147 / 310,47 \% \mathrm{PI})$, or moderate $(138 / 310$, $45 \%$ PI). It should be noted that the expert panel rated 22/
310 PI (7\%) with a major impact: 10 PI were related to anticoagulant treatment (enoxaparin, calciparin, or dabigatran) with either a DRP of duplication (4 PI), an absence of postoperative anticoagulation (2 PI), a non-conformity to guidelines (3 PI), or a supratherapeutic dosage (1 PI); 10 PI were related to the use of non-steroid anti-inflammatory medication (3 PI) or nefopam (7 PI) in older patients; and 


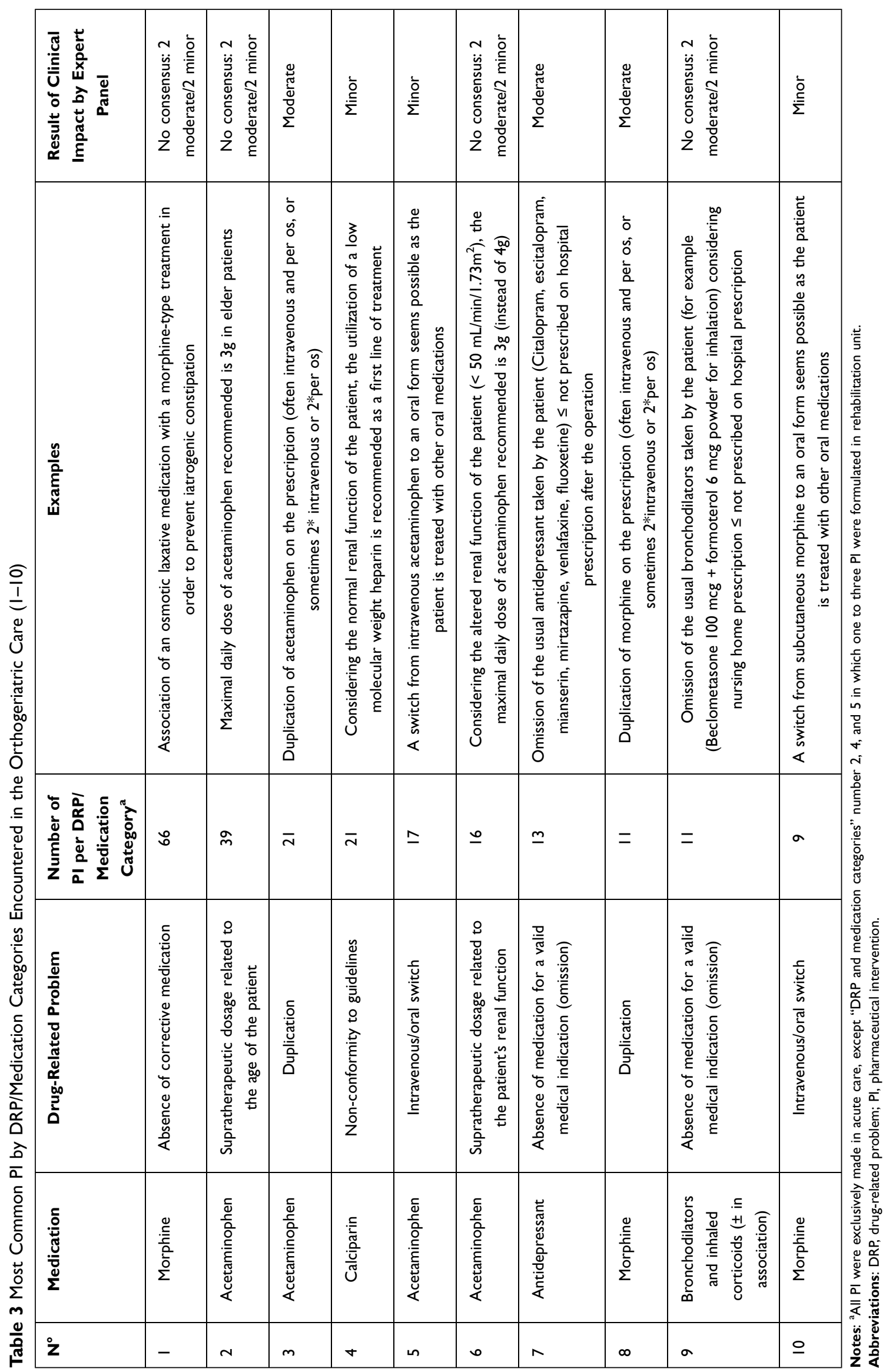




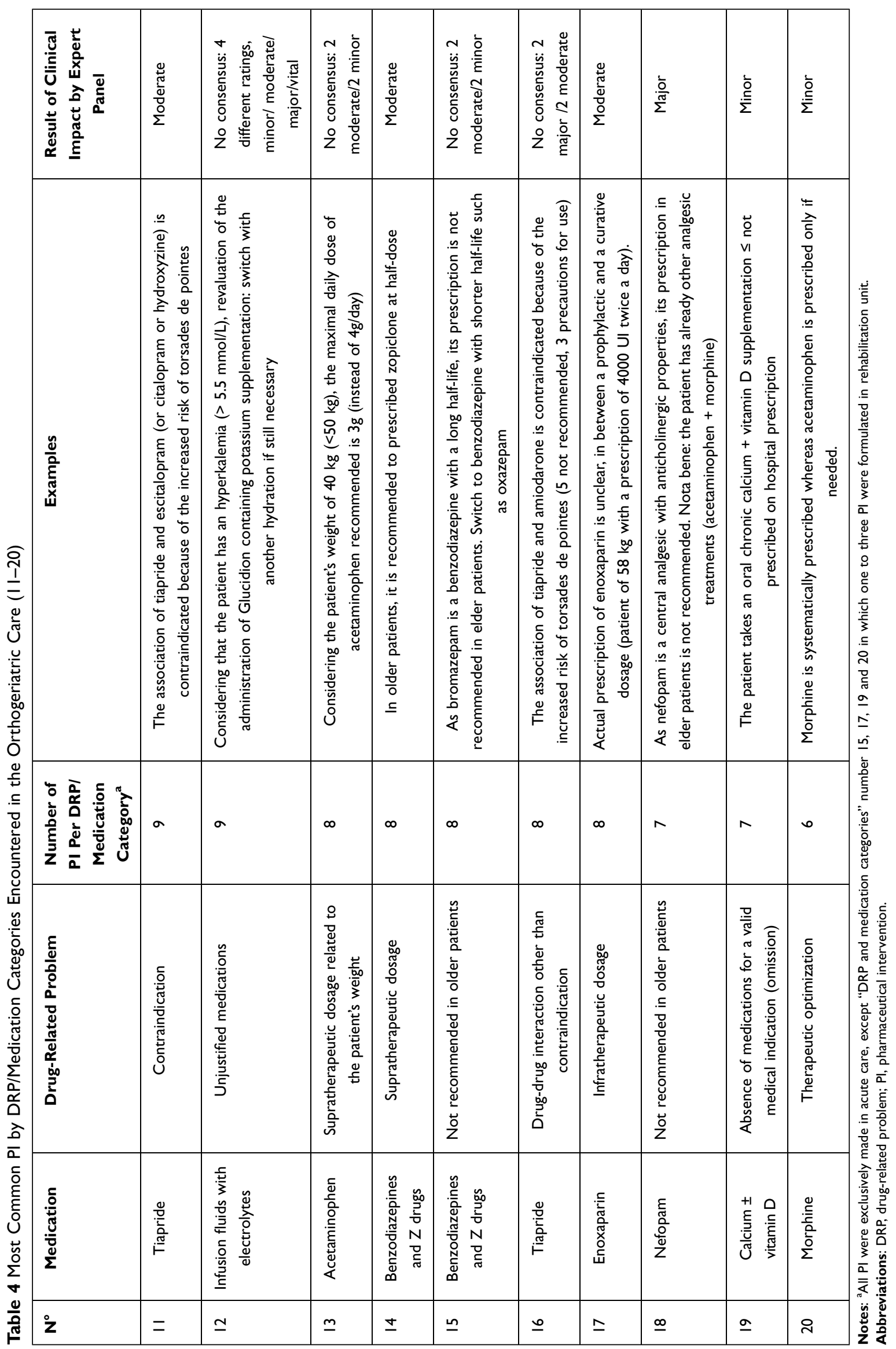


2 PI were related to metformin prescribed at a supratherapeutic dosage in patients with impaired renal function. Two PI were rated as having a vital impact (2/ $310,0.6 \% \mathrm{PI}$ ), which consisted in a contraindication of the association of digoxin with infusion fluids containing calcium ions (ringer lactate). One PI was rated as having a null clinical impact (1/310-0.3\% PI).

\section{Discussion}

Even though the implementation of clinical pharmacy activities was not fully reached, at least one potential ME was detected for almost three quarters of included patients. The results obtained herein are similar to previous reports in terms of potential ME per patient, either with PI or UID in geriatric or orthopaedic units. ${ }^{7,12,13,17,18}$ Moreover, the most frequent medication classes involved in PI in the present study (central nervous system, antithrombotic, and cardiovascular system medications) have also been found in studies led in orthopaedic units. ${ }^{19,20}$ However, among the central nervous system medication class, the proportion of analgesics compared to antidepressants was slightly different between acute care and rehabilitation facilities which illustrated the fact that PI made in orthopaedic units were different to the ones made in geriatric or rehabilitation units. Indeed, orthopaedic surgeons and anaesthetists do not revaluate the usual medications taken by the patient, because LOS in orthopaedic unit are often short. Consequently, some PI regarding revaluation of usual medications of patients were made exclusively in geriatric or rehabilitation units.

Furthermore, four "DRP and medication categories" almost exclusively made in acute care were of particular interest by the relative number of PI, despite the existing institution postoperative protocol for older patients: "absence of an osmotic laxative medication with a morphine-type treatment" and "supratherapeutic dosage of acetaminophen" either based on the patient's age, weight or renal function. Thereby, PI permitted to promote the use of this protocol in this specific population throughout our multi-site hospital, which seemed to be underused in acute care. Beyond $5 \mathrm{P}$ project, this protocol will be reviewed, regarding heparin and acetaminophen. The latter needed to be discussed in the context of the acute postoperative management of pain and the debate of reducing the dosage to $3 \mathrm{~g}$ per day only based on the patient's age. Henceforward, it will be important to raise awareness of this institutional protocol at each turnover of surgery or anaesthetist residents.
Most PI performed in the orthogeriatric care pathway were evaluated by the expert panel as having a significant clinical impact from improving patient's quality of life (almost one-third of PI rated as "minor") until preventing major or even vital damage that could have led to hospitalization or death. Similar results have been reported in a previous study ${ }^{19}$ using the same tool (CLEO), even if performed only in orthopaedic units with planned and unplanned surgeries, and rating only for a sample of $10 \%$ of PI. ${ }^{19}$ A strength of the present study is that the evaluation of the potential clinical impact of PI relied on a pluriprofessional and independent expert panel, which permitted to limit the interindividual variations that would have been induced by an evaluation performed by a single expert. No consensus was reached for almost half of PI, which highlights the importance of adapting PI to the step of the orthogeriatric care pathway. Indeed, the priorities of therapeutic management are slightly different between orthopaedic units (where the focus is made on pain and thromboprophylaxis management) and geriatric units including rehabilitation facilities (where a global reevaluation is performed). Further investigations on these differences of clinical impact rating should be conducted in order to increase the clinical relevance of pharmacists towards the prescriber, and so improve even more the quality of care of patients.

The present study has several limitations. Firstly, not all the patients benefitted from all the clinical pharmacy activities, therefore the number of potential ME may have been underestimated, especially in medication reconciliation at discharge. Indeed, an implementation study was conducted and a full-blown analysis of motives of non-realization is being performed. Moreover, according to Mekonnen et al, $^{21}$ medication reconciliation seems to be less effective during multiple transitions of care. So it could have been interesting to analyse whether the clinical impact of medication reconciliation was more important at discharge compared to transfer. A limitation of this study is that medication reconciliation at transfer (between units) and at discharge were not distinguished, which could explain the weak proportion of patients who had at least one UID at discharge. Furthermore, the potential clinical impact considered was evaluated only for PI related to prescription reviews, whereas it should also have been evaluated for UID to emphasise the relevance of clinical pharmacy 
activities. However, name of medications concerned by UID was not included in the initial data collection plan.

\section{Conclusions}

The present study showed that the patient personalised clinical pharmacy care programme permitted to secure the orthogeriatric care pathway by detecting a great number of potential ME, including PI mostly considered as having a significant clinical impact. Thus, the $5 \mathrm{P}$ project should be sustained as it permits to improve security and quality of patient therapeutic care throughout the multisite teaching hospital. Indeed, it required to coordinate our practices across units, better communicate between sites if patients were transferred, and enhance collaboration in daily routine between physicians, and with pharmacists and the paramedical teams. Regarding this positive impact on the securitisation of the therapeutic care, we could imagine to develop this new care pathway model of clinical pharmacy to other care pathways.

\section{Ethics Approval and Informed Consent}

This study was approved by the ethics committee of the Hospices Civils de Lyon on February, 26th 2019 and the Commission nationale de l'informatique et des libertés (CNIL, French national commission on data protection) following MR004 requirements $\mathrm{n}^{\circ} 18-314$ on May, 1st 2019.

\section{Acknowledgments}

The authors gratefully acknowledge all pharmacy students, pharmacy residents, pharmacists, and medical and paramedical professionals who participated to this study. 5P Study Group: GOUTELLE Sylvain, AUSSEDAT Marine, BOURGUIGNON Laurent, JANOLY-DUMENIL Audrey, BOLON-LARGER Magali, DODE Xavier, RANCHON Florence, DELANDE Evariste, CARPENTIER Isabelle, PIVOT Christine, MOUCHOUX Christelle, HOEGY Delphine, BARRAL Marine, MARTIN Julie, MANDY Bruno, RIOUFOL Catherine, DUSSART Claude, BREANT Valentine, LEBOUCHER Gilles, TOUZET Sandrine, DENIS Angélique, BOUVERET Laetitia.

\section{Author Contributions}

All authors made a significant contribution to the work reported, whether that is in the conception, study design, execution, acquisition of data, analysis and interpretation, or in all these areas; took part in drafting, revising or critically reviewing the article; gave final approval of the version to be published; have agreed on the journal to which the article has been submitted; and agree to be accountable for all aspects of the work.

\section{Funding}

This study was supported by a national public funding from the French Health ministry (winner of the 2017 national call for project in clinical pharmacy from the Direction Générale de l'Offre de Soins). It permitted to hire two full time pharmacists in charge of the implementation and achievement of pharmaceutical activities, and coordination of the $5 \mathrm{P}$ project.

\section{Disclosure}

The authors report no conflicts of interest in this work.

\section{References}

1. Veronese N, Maggi S. Epidemiology and social costs of hip fracture. Injury. 2018;49(8):1458-1460. doi:10.1016/j.injury.2018.04.015

2. Svedbom A, Hernlund E, Ivergård $\mathrm{M}$, et al. Osteoporosis in the European Union: a compendium of country-specific reports. Arch Osteoporos. 2013;8(1-2):1-2. doi:10.1007/s11657-013-0137-0

3. Prestmo A, Hagen G, Sletvold O, et al. Comprehensive geriatric care for patients with hip fractures: a prospective, randomised, controlled trial. Lancet. 2015;385(9978):1623-1633. doi:10.1016/S01406736(14)62409-0

4. Boddaert J, Cohen-Bittan J, Khiami F, et al. Postoperative admission to a dedicated geriatric unit decreases mortality in elderly patients with hip fracture. PLoS One. 2014;9(1):e83795. doi:10.1371/journal. pone. 0083795

5. Middleton M, Wan B, da Assunçao R. Improving hip fracture outcomes with integrated orthogeriatric care: a comparison between two accepted orthogeriatric models. Age Ageing. 2017;46(3):465-470. doi:10.1093/ageing/afw232

6. Kiesel E, Hopf Y. Hospital pharmacists working with geriatric patients in Europe: a systematic literature review. Eur J Hosp Pharm Sci Pract. 2018;25(e1):e74-e81. doi:10.1136/ejhpharm2017-001239

7. Beckett RD, Crank CW, Wehmeyer A. Effectiveness and feasibility of pharmacist-led admission medication reconciliation for geriatric patients. $J$ Pharm Pract. 2012;25(2):136-141. doi:10.1177/ 0897190011422605

8. Leguelinel-Blache G, Arnaud F, Bouvet S, et al. Impact of admission medication reconciliation performed by clinical pharmacists on medication safety. Eur J Intern Med. 2014;25(9):808-814. doi:10.1016/j. ejim.2014.09.012

9. Gizzi LA, Slain D, Hare JT, Sager R, Briggs F, Palmer CH. Assessment of a safety enhancement to the hospital medication reconciliation process for elderly patients. Am $J$ Geriatr Pharmacother. 2010;8(2):127-135. doi:10.1016/j.amjopha rm.2010.03.004

10. Wolf O, Åberg H, Tornberg U, Jonsson KB. Do orthogeriatric inpatients have a correct medication list? A pharmacist-led assessment of 254 patients in a Swedish University Hospital. Geriatr Orthop Surg Rehabil. 2016;7(1):18-22. doi:10.1177/2151458515625295 
11. Dooley MJ, Allen KM, Doecke CJ, et al. A prospective multicentre study of pharmacist initiated changes to drug therapy and patient management in acute care government funded hospitals. Br J Clin Pharmacol. 2004;57 (4):513-521. doi:10.1046/j.1365-2125.2003.02029.x

12. Cortejoso L, Dietz RA, Hofmann G, Gosch M, Sattler A. Impact of pharmacist interventions in older patients: a prospective study in a tertiary hospital in Germany. Clin Interv Aging. 2016;11:1343-1350. doi:10.2147/CIA.S109048

13. Donier L, Mondoloni P, Lory P, et al. Conciliation médicamenteuse priorisée sur les patients à risque en chirurgie programmée ou après admission par les urgences. [Priorization of medical reconciliation for patients at risk in scheduled surgery or after emergency admission]. EM-Consulte. 2019;54(2):137-144. doi:10.1016/j.phclin.2018.09.008

14. Hoegy D, Martin J, Barral M, et al. Development of clinical pharmacy programs integrated into patient care pathways using adverse event risks. Res Soc Adm Pharm. 2021. doi:10.1016/j. sapharm.2021.08.005

15. Laroche M-L, Charmes J-P, Merle L. Potentially inappropriate medications in the elderly: a French consensus panel list. Eur J Clin Pharmacol. 2007;63(8):725-731. doi:10.1007/s00228-007-0324-2

16. Vo TH, Bardet J-D, Charpiat B, et al. Validation of a tool for reporting pharmacists' interventions in everyday community pharmacy. J Clin Pharm Ther. 2018;43(2):240-248. doi:10.1111/jcpt.12642
17. Gillespie U, Alassaad A, Henrohn D, et al. A comprehensive pharmacist intervention to reduce morbidity in patients 80 years or older: a randomized controlled trial. Arch Intern Med. 2009;169 (9):894-900. doi:10.1001/archinternmed.2009.71

18. Hailu BY, Berhe DF, Gudina EK, Gidey K, Getachew M. Drug related problems in admitted geriatric patients: the impact of clinical pharmacist interventions. BMC Geriatr. 2020;20(1):13. doi:10.1186/ s12877-020-1413-7

19. Renaudin P, Coste A, Audurier Y, et al. Clinical, economic, and organizational impact of the clinical pharmacist in an orthopedic and trauma surgery department. J Patient Saf. 2018. doi:10.1097/ PTS.0000000000000539

20. Serandour N, Corbineau E, Langlois C, Waast D, Huon J-F. Evaluation of the incorporation of clinical pharmacists in a French orthopaedic surgery unit. J Pharm Clin. 2020;39(3):141-150. doi:10.1684/jpc.2020.0442

21. Mekonnen AB, McLachlan AJ, Brien J-AE. Effectiveness of pharmacist-led medication reconciliation programmes on clinical outcomes at hospital transitions: a systematic review and meta-analysis. BMJ Open. 2016;6(2):e010003. doi:10.1136/bmjopen-2015-010003
Clinical Interventions in Aging

\section{Publish your work in this journal}

Clinical Interventions in Aging is an international, peer-reviewed journal focusing on evidence-based reports on the value or lack thereof of treatments intended to prevent or delay the onset of maladaptive correlates of aging in human beings. This journal is indexed on PubMed Central, MedLine, CAS, Scopus and the Elsevier
Bibliographic databases. The manuscript management system is completely online and includes a very quick and fair peer-review system, which is all easy to use. Visit http://www.dovepress.com/ testimonials.php to read real quotes from published authors. 